same as at $B$, and as $B$ is on the boundary, this moment contributes to Poisson's Layer of surface density $\sigma$. If there are $n$ molecules per $\mathrm{cm}^{3}$ we have at once

$$
\sigma=\text { electric moment per unit volume }=n \alpha E .
$$

To relate this result to what is actually measured, namely, the drop in potential when the slab is introduced, we imagine a channel cut through the dielectric so that a unit positive charge may be taken from one plate to the other through it. If $E_{A}$ is the intensity before the dielectric is inserted, the force in the channel is $E_{A}-4 \pi \sigma$, and experiment shows that this must be assumed to be $E_{A} / K$, since the potential drops to $1 / K$ of its vacuum value, where $K$ is the dielectric constant. Thus :

$$
E=E_{A}-4 \pi \sigma=E_{A} / K=\sigma / n \alpha \text {, whence } K=1+4 \pi n \alpha .
$$

(2) If the molecules are supposed not uniformly polarized (and this must be the case on a microscopic view, since the polarizing force at $A$ must differ from that at $B$ owing to the different arrangement of surrounding molecules), then Poisson's volume distrihution of charge must be taken into account as well as the surface layers in order to calculate the force at the centre of a spherical cavity. This is never done, and it could not be done for lack of information as to how the polarization varies from point to point. The treatment which Clausius gave is therefore not valid.

The conclusion seems to be that only a macroscopic treatment can be applied and that the formula should loe

$$
K=1+4 \pi n \sigma .
$$

Thus for the molar polarization $P$ we should have

$$
P=\frac{K-1}{3}-\frac{M}{P}
$$

instead of Clausius-Mosotti

$$
P=\frac{K-}{\bar{K}+2} \cdot \frac{M}{\rho}
$$

( $M$ is molecular weight, $\rho$ is density).

$$
\text { G. Burniston Erown. }
$$

University College of London, at University College,

Bangor.

Nov. 8.

${ }^{1}$ Brown, G. B., Proc. Phys. Soc., 52, 577 (1940).

\section{Shape of Subjective Space}

THE apparent alteration in the size and shape and spatial relation of objects when right-eye and left-eye viewings are interchanged (or in other way the binocular parallax is reversed) may prove to some degree dependent on retinal structure, as suggested by Mr. Cox ${ }^{1}$, but considerable alterations of like character are certainly accounted for by peculiar geometrical conditions, in conjunction with extreme quickness of the eyes and mind to interpret these as normally as possible.

More than ten years ago, I directed general attention to the astonishing effect of viewing the Hyades or Orion's Belt, or similar bright star group, through a common inverting binocular, when the stars are behind a familiar object like the bare twigs and branches of a tree, or the figure of a man, a little way off. To most observers with normal sight the stars appear in the air half-way to the tree, which in some cases may mean only a yard away through the bunoculars. That provides or points out the general illustrative kind of case for such phenomena, even for the naked eyes, though the effects are rather feebly noticeable at long distances when telescopes are not used.

Briefly, explanations of the half-way appearance may be arranged like this: The distance $d$ of the tree is assessed at once, in the ordinary view, by convergence (so far as the distance is short) and by the angular subtense which the tree shows as a chosen familiar object. After the reversal of parallax there is no natural convergence at all to use (only eyestraining divergence), but most observers still assess d. well enough by the angular subtense. The ordinary relative parallax of the tree and heavens is like $\frac{1}{d}-\frac{1}{\infty}$. After reversal it is similarly like $\frac{1}{x}-\frac{1}{d}$, where $x$ is the apparent distance of the heavens, a distance now less than $d$ because of the reversal. Of course $\frac{1}{x}-\frac{1}{d}=\frac{1}{d}-\frac{1}{\infty}$, so $x=d / 2$, and that the eyes and mind instantly recognize, after their lifelong experience of similar parallax problems. Only to the extent the object is not familiar, or not single and distinct but confused, may the observer not promptly recognize $d$, and then it is that the heavens are found reluctant to leave their usual remoteness. The telescopes of magnification $m$ make $x$ as small as $d / 2 m$; and they also aid by their ordinary telescopic flattening of the tree. The interchange of the far and near parts of the tree is curious but not very important. When the sun (or moon) is in view with the tree, the linear diameter of the sun appears as small as $d / 230$ feet, which is sometimes as little as two or three inches. Intrusive twigs make a transparency effect.

It is evident that the subjective spatial change in this example is remarkable. The whole universe has in effect been turned inside out at the dictate of the tree and its scale-fixing distance $d$. The outermost parts towards infinity have been brought in to a spherical surface of only $d / 2 m$ ft. radius around the observer, and parts nearest the observer have been dispersed towards infinity. Accordingly, to some observers in clear air over a featureless plain, dozens of men ranged in line from as near as the tree, outwardly to the horizon, appear as dwarfs near at hand tapering outwards to become giants in the distance. Perhaps because the dwarfs are more crowded, numerous and uninteresting, normality usually seems allotted to them, and magnification is declared the chief impression. Possibly causes of similar kind might be found involved in some of the magnification effects which have come under Mr. Cox's notice.

The mind's instant, unhesitating use of the eyes and former experiences, or memory, as instruments in these subjective phenomena is noteworthy. The geometry and the eyes cannot prove the whole of the interest. In that connexion, regard should certainly be had to Sir Richard Paget's communication in NATURE of July 22, 1939, p. 153.

58 Raleigh Road,

S. L. WALKOEN.

London, N.8. Nov. 16.

${ }^{1}$ NAtURe, 150, 349 (1942). 